\title{
THE LOWER BOUND OF THE MAXIMAL DILATATION OF THE BEURLING-AHLFORS EXTENSION
}

\section{Li Zhong}

\section{Introduction}

An increasing continuous function $h$ defined on an interval $I \subset \mathbf{R}^{1}$ is $\varrho$ quasisymmetric on $I$ if

$$
\varrho^{-1} \leq \frac{h(x+t)-h(x)}{h(x)-h(x-t)} \leq \varrho
$$

for all $x$ and $t>0$ such that $[x-t, x+t] \subset I$.

A well-known result due to Beurling and Ahlfors [1] states that the map $f_{h, r}$ defined by

$$
f_{h, r}(z)=\frac{1}{2}[\alpha(z)+\beta(z)+i r(\alpha(z)-\beta(z))]
$$

where $r>0$,

$$
\alpha(z)=\int_{0}^{1} h(x+y t) d t, \quad \beta(z)=\int_{-1}^{0} h(x-y t) d t, \quad z=x+i y,
$$

is a quasiconformal extension of $h$ to the upper half-plane $H$ if $h$ is $\varrho$-quasisymmetric on $\mathbf{R}^{1}$. Such a map $f_{h, r}: H \rightarrow H$ is called a Beurling-Ahlfors extension of $h$. Beurling and Ahlfors proved that if $h$ is $\varrho$-quasisymmetric on $\mathbf{R}^{1}$, there is a number $r>0$ such that the maximal dilatation $K\left[f_{h, r}\right] \leq \varrho^{2}$. This estimation has been replaced by

$$
K\left[f_{h, 1}\right] \leq 8 \varrho, \quad K\left[f_{h, 1}\right] \leq 4.2 \varrho, \quad \text { and } \quad K\left[f_{h, 1}\right] \leq 2 \varrho
$$

due to T. Reed [8], Li Zhong [7] and M. Lehtinen [3], respectively. M. Lehtinen [4] even proved that $K\left[f_{h, r}\right] \leq 2 \varrho-1$ for some $r>0$.

In this paper, the lower bound of $K\left[f_{h, r}\right]$ will be examined. We denote

$$
K_{\varrho}:=\sup _{h \in S_{\varrho}}\left\{\inf _{r>0} K\left[f_{h, r}\right]\right\},
$$

where $S_{\varrho}$ is the set of all $\varrho$-quasisymmetric functions on $\mathbf{R}^{1}$. We shall give an example of a $\varrho$-quasisymmetric function $h$ such that

$$
K\left[f_{h, r}\right] \geq(2 \varrho+1)\left(1-\frac{1}{\sqrt{\varrho}}\right)
$$

for every $r>0$. This implies the following theorem. 
Theorem. We have $K_{\varrho} \geq(2 \varrho+1)(1-1 / \sqrt{\varrho})$ for every $\varrho \geq 1$.

This result tells us that the coefficient of $\varrho$ in a linear upper bound of $K\left[f_{h, r}\right]$ generally cannot be smaller than 2 . This means that the above results by Lehtinen are sharp in a certain sense.

By this theorem and the results of Lehtinen, we have

Corollary. We have $\lim _{\varrho \rightarrow \infty} K_{\varrho} / \varrho=2$.

\section{Piecewise linear quasisymmetric functions}

To give a special $\varrho$-quasisymmetric function, we need some lemmas on piecewise linear quasisymmetric functions.

Lemma 1. Let $E \subset[0,1],\{0,1\} \subset E$, be a set of finite points and $h:[0,1] \rightarrow$ $[0,1], h(0)=0, h(1)=1$, be increasing and continuous on $[0,1]$ and linear on each interval in $[0,1] \backslash E$. If (1) is true for all $x$ and $t>0$ such that $\{x-t, x, x+t\} \cap E$ has at least two points, then $h$ is $\varrho$-quasisymmetric on $[0,1]$.

This lemma is proved by Hayman and Hinkkanen ([2]).

Noting that $h$ is $\varrho$-quasisymmetric if and only if $f \circ h \circ g$ is $\varrho$-quasisymmetric when $f$ and $g$ are increasing linear functions, Lemma 1 can easily be generalized to the following statement:

Lemma $1^{\prime}$. Let $E \subset[a, b],\{a, b\} \subset E$, be a set of finite points and $h:[a, b] \rightarrow[c, d], h(a)=c, h(b)=d$, be increasing and continuous on $[a, b]$ and linear on each interval in $[a, b] \backslash E$. If (1) is true for all $x$ and $t>0$ such that $\{x-t, x, x+t\} \cap E$ has at least two points, then $h$ is $\varrho$-quasisymmetric on $[a, b]$.

Lemma 2. Let $E \subset \mathbf{R}^{1}$ be a set of $n$ points and $h: \mathbf{R}^{1} \rightarrow \mathbf{R}^{1}$ be increasing and continuous on $\mathbf{R}^{1}$ and linear on each interval in $\mathbf{R}^{1} \backslash E$. Suppose that (1) is true for all $x$ and $t>0$ such that $\{x-t, x, x+t\} \cap E$ has at least two points and

$$
\varrho^{-1} \leq \lim _{x \rightarrow+\infty} \frac{h(x)}{-h(-x)} \leq \varrho
$$

Then $h$ is $\varrho$-quasisymmetric on $\mathbf{R}^{1}$.

Proof. Without any loss of generality, we may assume that $n \geq 2$. For if $n=1$, the condition (4) implies that $h$ is $\varrho$-quasisymmetric on $\mathbf{R}^{1}$. Suppose that $E=\left\{x_{1}, x_{2}, \ldots, x_{n}\right\}$ with $x_{1}<x_{2}<\cdots<x_{n}$. Let $A$ be a sufficiently large number and $E^{\prime}=E \cup\left\{-A, A+2 x_{1}\right\}$.

To prove that $h$ is $\varrho$-quasisymmetric on $\mathbf{R}^{1}$, it is sufficient to show that $h$ is $\varrho$-quasisymmetric on $\left[-A, A+2 x_{1}\right]$ for any sufficiently large $A$. By Lemma $1^{\prime}$, we should only check whether (1) is true for all $x$ and $t>0$ such that $\{x-t, x$, $x+t\} \cap E^{\prime}$ has at least two points. But we have supposed that (1) is true for all $x$ 
and $t>0$ such that $\{x-t, x, x+t\} \cap E$ has at least two points. So it is sufficient to show that

$$
\varrho^{-1} \leq \frac{h\left(A+2 x_{1}\right)-h\left(x_{1}\right)}{h\left(x_{1}\right)-h(-A)} \leq \varrho
$$

$$
\varrho^{-1} \leq \frac{h\left(A+2 x_{1}\right)-h\left(x_{j}\right)}{h\left(x_{j}\right)-h\left(2 x_{j}-A-2 x_{1}\right)} \leq \varrho
$$

and

$$
\varrho^{-1} \leq \frac{h\left(A+2 x_{j}\right)-h\left(x_{j}\right)}{h\left(x_{j}\right)-h(-A)} \leq \varrho
$$

for all $j=2, \ldots, n$.

For any given $x_{j} \in E$, we look at the function $\varphi_{j}(t)=\left[h\left(x_{j}+t\right)-\right.$ $\left.h\left(x_{j}\right)\right] /\left[h\left(x_{j}\right)-h\left(x_{j}-t\right)\right]$. Obviously, when $t>\tau_{j}=\max \left\{\left|x_{l}-x_{j}\right| \mid l=1, \ldots, n\right\}$, $\varphi_{j}^{\prime}(t)$ keeps its sign. Hence if $\varphi_{j}^{\prime}(t)>0$ as $t>\tau_{j}$,

$$
\varphi_{j}\left(\tau_{j}\right) \leq \frac{h\left(x_{j}+t\right)-h\left(x_{j}\right)}{h\left(x_{j}\right)-h\left(x_{j}-t\right)} \leq \lim _{n \rightarrow+\infty} \varphi_{j}(\eta)
$$

and if $\varphi_{j}^{\prime}(t)<0$ as $t>\tau_{j}$

$$
\lim _{\eta \rightarrow+\infty} \varphi_{j}(\eta) \leq \frac{h\left(x_{j}+t\right)-h\left(x_{j}\right)}{h\left(x_{j}\right)-h\left(x_{j}-t\right)} \leq \varphi_{j}\left(\tau_{j}\right)
$$

for $j=1,2, \ldots, n$. Since $h$ is increasing and linear on $\left(x_{n}, \infty\right)$ and $\left(-\infty, x_{1}\right)$, $h(x) \rightarrow+\infty$ as $x \rightarrow+\infty$ and $h(x) \rightarrow-\infty$ as $x \rightarrow-\infty$. Hence

$$
\lim _{\eta \rightarrow+\infty} \varphi_{j}(\eta)=\lim _{x \rightarrow+\infty} \frac{h(x)}{-h(-x)}
$$

for $j=1,2 \ldots, n$. By (4) we have

$$
\varrho^{-1} \leq \lim _{\eta \rightarrow+\infty} \varphi_{j}(\eta) \leq \varrho, \quad j=1,2, \ldots, n
$$

From (8), (9) and (10) we see that if

$$
\varrho^{-1} \leq \varphi_{j}\left(\tau_{j}\right) \leq \varrho, \quad j=1,2, \ldots, n,
$$

then

$$
\varrho^{-1} \leq \frac{h\left(x_{j}+t\right)-h\left(x_{j}\right)}{h\left(x_{j}\right)-h\left(x_{j}-t\right)} \leq \varrho, \quad j=1,2, \ldots, n
$$


for $t>\max \left\{\tau_{1}, \tau_{2}, \ldots, \tau_{n}\right\}$, and hence (5), (6), and (7) hold. It remains to prove (11).

Since $n>1, \tau_{j}$ is positive for $j=1,2, \ldots, n$. Then we see that $\left\{x_{j}-\right.$ $\left.\tau_{j}, x_{j}, x_{j}+\tau_{j}\right\} \cap E$ has at least two points. By the assumption of the lemma, (11) is true. The lemma is proved.

For any $s \geq 1$, we define a function $h_{s}$ as follows:

$$
h_{s}(x):= \begin{cases}1+s(x-1) & \text { as } x \geq 1 \\ s(1+s)^{-1}+s^{-1}\left(x-(1+s)^{-1}\right) & \text { as }(1+s)^{-1} \leq x \leq 1, \\ s x & \text { as }-s(1+s)^{-1} \leq x \leq(s+1)^{-1} \\ -s^{2}(1+s)^{-1}+s^{3}\left(x+s(1+s)^{-1}\right) & \text { as }-1 \leq x \leq-s(1+s)^{-1} \\ -s^{2}+s(x+1) & \text { as } x \leq-1 .\end{cases}
$$

We are now going to show that $h_{s}$ is an $s^{2}$-quasisymmetric function. This quasisymmetric function will be used to prove the main theorem in the next paragraph.

Let $E=\left\{-1,-s(1+s)^{-1},(1+s)^{-1}, 1\right\}$. Obviously, there are $3 \times C_{4}^{2}=18$ cases in each of which $\{x-t, x, x+t\} \cap E$ has at least two points. We omit three cases $\{x-t, x, x+t\}$ that are on the same interval in $\mathbf{R}^{1} \backslash E$. For all remaining cases, one may check (1) directly by simple computation. By $\Delta$ we denote $\left[h_{s}(x+t)-h_{s}(x)\right] /\left[h_{s}(x)-h_{s}(x-t)\right]$. Then we have

Case 1: $x=(1+s)^{-1}, x+t=1$. Then $\Delta=s^{-2}$.

Case 2: $x-t=(1+s)^{-1}, x=1$. Then $\Delta=s$.

Case 3: $x=-s(1+s)^{-1}, x+t=1$. Then $\Delta=\left(s^{2}+s+1\right) /\left(s^{3}+2 s\right)$ and $s^{-2} \leq \Delta \leq 1$.

Case 4: $x-t=-s(1+s)^{-1}, x+t=1$. Then $\Delta=(s+2) /\left(2 s^{2}+s\right)$ and $s^{-2} \leq \Delta \leq 1$.

Case 5: $x-t=-s(1+s)^{-1}, x=1$. Then $\Delta=(2 s+1) /\left(s^{2}+s+1\right)$ and $s^{-1} \leq \Delta \leq 1$.

Case 6: $x=-1, x+t=1$. Then $\Delta=\left(s^{2}+1\right) / 2 s$ and $1 \leq \Delta \leq s$.

Case 7: $x-t=-1, x+t=1$. Then $\Delta=s^{-2}$.

Case 8: $x-t=-1, x=1$. Then $\Delta=2 s /\left(s^{2}+1\right)$ and $s^{-1} \leq \Delta \leq 1$.

Case 9: $x=-s(1+s)^{-1}, x+t=(1+s)^{-1}$. Then $\Delta=s^{-1}$.

Case 10: $x-t=-s(1+s)^{-1}, x=(1+s)^{-1}$. Then $\Delta=s^{-2}$.

Case 11: $x=-1, x+t=(1+s)^{-1}$. Then $\Delta=\left(s^{2}+s+1\right) /(s+2)$ and $1 \leq \Delta \leq s$.

Case 12: $x-t=-1, x+t=(1-s)^{-1}$. Then $\Delta=(s+2) /\left(2 s^{2}+s\right)$ and $s^{-2} \leq \Delta \leq 1$.

Case 13: $x-t=-1, x=(1+s)^{-1}$. Then $\Delta=s^{2}$. 
Case 14: $x=-1, x+t=-s(1+s)^{-1}$. Then $\Delta=s^{2}$.

Case 15: $x-t=-1, x=-s(1+s)^{-1}$. Then $\Delta=s^{-2}$.

Therefore we have

$$
s^{-2} \leq \frac{h_{s}(x+t)-h_{s}(x)}{h_{s}(x)-h_{s}(x-t)} \leq s^{2} \quad \text { for cases 1) - 15). }
$$

Moreover, we easily see that

$$
\lim _{x \rightarrow+\infty} \frac{h_{s}(x)}{-h_{s}(-x)}=1
$$

From (13) and (14), we can conclude by Lemma 2 that $h_{s}$ is $s^{2}$-quasisymmetric on $\mathbf{R}^{1}$.

\section{The proof of the main result}

The quasisymmetric function $h_{s}$ constructed in the previous paragraph has some special properties. Obviously,

$$
h_{s}(0)=0, \quad h_{s}(1)=1, \quad h_{s}(-1)=-s^{2} .
$$

By a simple computation, we get

$$
\int_{0}^{1} h_{s}(t) d t=\frac{s}{s+1}, \quad \int_{-1}^{0} h_{s}(t) d t=-\frac{s^{2}}{1+s} .
$$

Using these properties one obtains a lower estimate of $K_{\varrho}$.

We denote $h_{s}$ by $h$ and $s^{2}$ by $\varrho$. Then $h$ is a $\varrho$-quasisymemtric function on $\mathbf{R}^{1}$. Let $f_{r, h}$ be the Beurling-Ahlfors extension of $h$. The dilatation of $f_{h, r}$ at $i$ is denoted by $D_{r}$. Setting $\xi=\alpha_{y}(i) / \alpha_{x}(i), \eta=-\beta_{y}(i) / \beta_{x}(i), \zeta=\alpha_{x}(i) / \beta_{x}(i)$, we get

$$
D_{r}+D_{r}^{-1}=a(\xi, \eta, \zeta) r+b(\xi, \eta, \zeta) / r
$$

where

$$
\begin{aligned}
& a(\xi, \eta, \zeta)=\left[(\zeta-1)^{2}+(\zeta \xi+\eta)^{2}\right] /[2 \zeta(\xi+\eta)] \\
& b(\xi, \eta, \zeta)=\left[(\zeta+1)^{2}+(\zeta \xi-\eta)^{2}\right] /[2 \zeta(\xi+\eta)]
\end{aligned}
$$

From (15) and (16) one obtains

$$
\zeta=-1 / h(-1)=s^{-2},
$$




$$
\begin{gathered}
\xi=1-\int_{0}^{1} h(t) d t=(1+s)^{-1} \\
\eta=1+\zeta \int_{-1}^{0} h(t) d t=\frac{s}{1+s}
\end{gathered}
$$

Hence we have

$$
D_{r}+D_{r}^{-1} \geq(a(\xi, \eta, \zeta) \cdot b(\xi, \eta, \zeta))^{1 / 2}
$$

$$
\begin{aligned}
& =\frac{1}{\zeta(\xi+\eta)}\left(\left((\zeta-1)^{2}+(\zeta \xi+\eta)^{2}\right)\left((\zeta+1)^{2}+(\zeta \xi-\eta)^{2}\right)\right)^{1 / 2} \\
& =s^{2}\left(\left(\left(\frac{1}{s^{2}}-1\right)^{2}+\left(\frac{1}{s^{2}(s+1)}+\frac{s}{s+1}\right)^{2}\right)\left(\left(\frac{1}{s^{2}}+1\right)^{2}+\left(\frac{1}{s^{2}(1+s)}-\frac{s}{1+s}\right)^{2}\right)\right)^{1 / 2} \\
& =\frac{1}{s^{2}}\left(\left(\left(s^{2}-1\right)^{2}+\left(\frac{1+s^{3}}{1+s}\right)^{2}\right)\left(\left(s^{2}+1\right)^{2}+\left(\frac{s^{3}-1}{1+s}\right)^{2}\right)\right)^{1 / 2} .
\end{aligned}
$$

Noting that

one obtains

$$
\frac{s^{3}-1}{s+1} \geq s^{2}-s \quad \text { and } \quad \frac{s^{3}+1}{s+1}=s^{2}-s+1
$$

and hence

$$
\begin{aligned}
D & +D^{-1} \geq \frac{1}{s^{2}}\left(\left(2 s^{4}-2 s^{3}+s^{2}-2 s+2\right)\left(2 s^{4}-2 s^{3}+3 s^{2}+1\right)\right)^{1 / 2} \\
& =\frac{1}{s^{2}}\left(4 s^{8}-8 s^{7}+12 s^{6}-12 s^{5}+13 s^{4}-12 s^{3}+7 s^{2}-2 s+2\right)^{1 / 2} \\
& =\frac{1}{s^{2}}\left(\left(2 s^{4}-2 s^{3}+2 s^{2}-s\right)^{2}+5 s^{4}-8 s^{3}+6 s^{2}-2 s+2\right)^{1 / 2} .
\end{aligned}
$$

Setting $P(s)=5 s^{4}-8 s^{3}+6 s^{2}-2 s+2$, one computes

$$
P(1)=3>0, \quad P^{\prime}(1)=6>0, \quad P^{\prime \prime}(s)=60 s^{3}-48 s+12 \geq 0 \text { as } s \geq 1,
$$

and hence $P(s)>0$ as $s \geq 1$. Then we have

$$
D+D^{-1}>\frac{1}{s^{2}}\left(2 s^{4}-2 s^{3}+2 s^{2}-s\right)=2 s^{2}-2 s+2-\frac{1}{s} .
$$

Since $D^{-1} \leq 1$, we immediately obtain

$$
D>2 s^{2}-2 s+1-s^{-1}=\left(2 s^{2}+1\right)\left(1-s^{-1}\right) \text {. }
$$

Replacing $s$ by $\sqrt{\varrho}$, we get

$$
D>(2 \varrho+1)(1-1 / \sqrt{\varrho})
$$

and $K_{\varrho}>(2 \varrho+1)(1-1 / \sqrt{\varrho})$. The main theorem is proved. 
Some remarks: 1. The author suggested another piecewise linear quasisymmetric function on $\mathbf{R}^{1}$ which is similar to $h_{s}$ in this paper but more complicated. $\mathrm{Li}$ Wei and Liu Yong computed the maximal dilatation of its Beurling-Ahlfors extension ([6]) and got an asymptotic estimate.

2. There are some other results on the lower bound of $K_{\varrho}$. For instance, $K_{\varrho} \geq 1.587 \varrho$ for large $\varrho([7]) ; K_{\varrho} \geq 3 \varrho / 2$ for every $\varrho \geq 1$ and $\lim _{\varrho \rightarrow+\infty} K_{\varrho} / \varrho \geq$ $1.5625([4]) ; K_{\varrho}>8 \varrho / 5$ for $\varrho>7$ and $\lim _{\varrho \rightarrow+\infty} K_{\varrho} / \varrho \geq 1.765625$ ([5]).

\section{References}

[1] Beurling, A., and L.V. AhLFors: The boundary correspondence under quasiconformal mappings. - Acta Math. 96, 1956, 125-142.

[2] Hayman, W., and A. HinkKanen: Distortion estimates for quasisymmetric functions. Ann. Univ. Mariae Curie-Sklodowska Sect. A 36-37, 1982, 51-67.

[3] LEHTinen, M.: The dilatation of Beurling-Ahlfors extensions of quasisymmetric functions. - Ann. Acad. Sci. Fenn. Ser. A I Math. 8, 1983, 187-192.

[4] Lehtinen, M.: Remarks on maximal dilatation of the Beurling-Ahlfors extension. - Ann. Acad. Sci. Fenn. Ser. A I Math. 9, 1984, 133-139.

[5] Lehtinen, M.: On maximal and minimal quasisymmetric functions on an interval. - Ann. Acad. Sci. Fenn. Ser. A I Math. 12, 1987, 77-84.

[6] LI WEI and LIU YoNG: On dilatation of the Beurling-Ahlfors extension of a quasisymmetric function. - Adv.in Math. 15, 1986, 419-426 (Chinese).

[7] Li ZhoNG: On the Beurling-Ahlfors extension. - Acta Math. Sinica 26, 1983, 279-290 (Chinese).

[8] REeD, T.: Quasiconformal mappings with given boundary values. - Duke Math. J. 33, $1966,459-464$.

Peking University

Mathematics Department

Beijing 100871

China

Received 22 February 1989 